\title{
Local Minimum Energy of LBP Algorithm Concrete CT Image Segmentation
}

\author{
Liang Zhao ${ }^{1}$, Sheng-Jun $\mathrm{Xu}{ }^{1}$, Jun $\mathrm{Lu}^{2}$ and Deng-Feng Chen ${ }^{1}$ \\ ${ }^{1}$ Dept of Info and Automation, Xi'an Univ. Of Arch. \& Tech. Xi'an 710055, China \\ ${ }^{2}$ School of Automation and Information Engineering,Xi'an University of \\ Technology, Xi'an 710048, China \\ zhaoliang@xauat.edu.cn
}

\begin{abstract}
Concrete CT image segmentation is a hot civil engineering research field in recent years. An algorithm of minimizing the energy of local region is proposed to solve the problem of interaction fails to capture statistical property of natural image and to segment concrete CT image. At first, the algorithm utilizes local region information of image to construct the energy model of local region, establishes a segmentation model of local interaction region based on MRF. Then using loopy belief propagation (LBP) algorithm and other algorithms optimizes global energy. In process of optimization, local region energy is converged, and the label of local region is estimated based on MAP algorithm. Then the information of local region is transferred to the region of neighborhood. The result of experiment shows that comparing with standard LBP algorithm, the new algorithm has a better segmentation result, and efficiently restrained effect on image noise and texture for segmentation. Result of concrete CT image segmentation will simplify the following CT statistical analysis and provide an important method for reaching real meso-structure of concrete's finite element network.
\end{abstract}

Keywords: concrete CT image, image segmentation, local region energy, Markov Random Field (MRF)

\section{Introduction}

Image segmentation is one of the fundamental questions in image processing. Precise and quick Segmentation is most important on the analysis and understanding of more complex image [1]. In recent years, the image processing technology based on MRF model has developed rapidly, but the Pair-wise MRF model cannot fully describe the affluently statistical property of natural image [2]. If we adopt high-level MRF model, it will lead to a huge calculation. It is hard to satisfy the practical demands. Recently, local image region and small region of image are often used to build the model of local structure, and to take the place of pairs-pixel of image [3, 4]. This kind of MRF model based on local structure can reduce the calculation of traditionally MRF segmentation strategy in some ways, while it can extract the information of structure at the same time [5]. Chen proposed the HOPS (higher order proxy neighborhoods) model based on Pair-wise MRF to establish high order neighborhood [6]. Wang proposed that using SMRF (Super pixel MRF) model to establish the relationship in local region of image [7]. Jia proposed a similar model, which presegments the image by adopting Mcan Shift algorithm [8]. Then according to the result of segmentation, it established the model of neighborhood region, and got the final segmentation result by iteration with the adopting of LBP algorithm. 
However, slow convergence is still a problem on algorithms. And segmentation algorithm is easily influenced by the result of pre-segmentation in reference [7-8]. So, how to choose the parameter in pre-segmentation algorithm is still an important question, and based on the region model is easily produce fake boundaries between the segmentation regions [9]. Based on the Pairwise MRF model, a new algorithm based on minimizing energy of local region is proposed. It establishes a local interaction region based on MRF segmentation model. According to regional information transferred, a more efficiently accelerating strategy is proposed. The experiment showed that a fine segmentation result is received of the algorithm, and efficiently restrained effect on image noise and texture for segmentation. Result of concrete CT image segmentation experiment can better classified aggregate region, mortar region and cracks region. And the result will simplify the following CT statistical analysis and provide an important method for reaching real meso-structure of concrete's finite element network.

\section{Image Segmentation Energy Model}

\subsection{Region Segmentation MRF Model}

MRF has been used widely in image aspects of extracting boundaries, segmenting, and analyzing texture. In image processing can be summarized as label problem of image. Figure 1 is a MRF model.

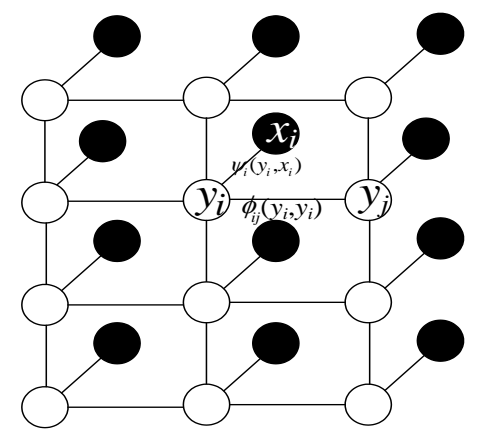

Figure 1. MRF Model

A two-dimension image can be seen $X=\left\{x_{1}, x_{2}, \cdots, x_{N}\right\} \cdot Y=\left\{y_{1}, y_{2}, y_{3}, \ldots, y_{N}\right\}$ is the segmentation result of image. And $y$ is the label for pixel $i, y_{i} \in\{1,2,3, \ldots, L\}, L$ is the label number of observing image. According system point neighborhood establishes relation, $C_{x}=\{C: x \in X\}$. The label problem of observing image is described by MRF model. According to the HAMMERSLEY-CLIFFORD theory [10], MRF and Gibbs Random Field have consistency. So we can confirm MRF field by the establishment of Gibbs Random Field. And the prior probability of label field Y:

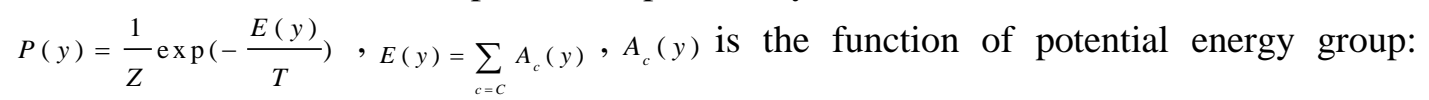
$A_{c}(y)=A_{c}{ }^{\prime}\left(y_{i}, y_{j}\right)=\left\{\begin{array}{ll}-\beta, & y_{i}=y_{j} \\ \beta, & y_{i} \neq y_{j}\end{array}, \quad\right.$ is segmentation parameter of potentials which controls consistency of region. $\mathrm{Z}$ is normalization function.

For observing image $\mathrm{X}$, define local region $l_{i}$ with $w \times w$. So the observing image $X$ can be distributed sub graph of overlap $W \times H . W$ Is width of image and $H$ is height of image. 
Supposing the pixels of image just correspond to the label of condition Gaussian distribution, and supposing the condition Gaussian distribution of two pixels is independently distributed,

$$
P(X \mid Y, \theta)=\prod_{i \in l_{i}} p\left(x_{i} \mid y_{i}, \theta\right)=\prod_{i \in l_{i}} \frac{1}{\sqrt{2 \pi} \sigma_{i}} \exp \left(-\frac{\left(y_{i}-\mu_{i}\right)^{2}}{2 \sigma_{i}^{2}}\right)
$$

In (1), $\theta=\left(\mu_{1}, \sigma_{1}, \mu_{2}, \sigma_{2}, \ldots \mu_{k}, \sigma_{k}, \ldots, \mu_{M}, \sigma_{M}\right), \mu_{k}, \sigma_{k}$ is gauss mean value and variance of $\mathrm{k}$. In this paper, we use expectation maximization (EM) to estimate parameter $\left(\mu_{k}, \sigma_{k}\right)$. EM is widely applied on parameter estimation of imperfect data problem, the basic estimation processes as follows:

Expectation Step: to evaluate expectation value of $Q\left(\theta, \theta^{p}\right)$ on full data after confirming model.

$$
Q\left(\theta, \theta^{p}\right)=E\left[\log P(X, Y \mid \theta) \mid X, \theta^{p}\right]
$$

$\theta^{p}$ Is parameter of iterating estimation in $P$ times? $\theta=\left\{\left(\alpha_{k}, \mu_{k}, \sigma_{k}\right), k=1.2 \ldots, L\right\}$ Is a new parameter.

Maximization Step: to evaluate new estimated value of parameter $\theta^{p+1}$ after maximizing $Q\left(\theta, \theta^{p}\right)$

$$
\theta^{p+1}=\underset{\theta}{\arg \max } E\left[\log P(X, Y \mid \theta) \mid X, \theta^{p}\right]
$$

Return to step E, re-estimate expectation of $Q\left(\theta, \theta^{p}\right)$, and keep iterating until convergence, get the final estimation of parameter $\theta^{p}$.

\subsection{Energy Segmentation Model}

In MRF, the distribution problem of label number can always be summed up as a minimizing problem of MRF global energy. Recently, partitioning technology is often used to establish local region image model. But the local region model which is Non-Overlapping always produces new fake boundaries among the local region of image. According to Pairwise MRF model, this paper will adopt local interaction region MRF energy model.

Based on local region MRF model in chapter 2.1, we defined a local energy model based on the pixel $\mathrm{x}$ regarded as the centre point of local region $l_{i}$ :

$$
E_{l_{i}}(x)=\sum_{i \in l_{i}} E_{i}\left(x_{i}\right)+\sum_{\forall i j \in l_{i}} E_{i j}\left(x_{i}, x_{j}\right)
$$

$\sum_{i \in l_{i}} E_{i}\left(x_{i}\right)$ is likelihood energy of local region $l_{i}$. It represents the likelihood energy sum of distributing a label to each pixel $x_{i}$ in a local region $l_{i}, \sum_{i \in l_{i}} E_{i}\left(x_{i}\right)=\sum_{i \in l_{i}} E_{i}\left(x_{i}, y_{i}, \theta\right)$, $\sum_{\forall i j \in l_{i}} E_{i j}\left(x_{i}, x_{j}\right)$ is smooth energy in local region, it represents the total energy of distributing two labels on $i j$ from all the neighborhood pixel of local region $l_{i}$, $\sum_{\forall i j \in l_{i}} E_{i j}\left(x_{i}, x_{j}\right)=\sum_{i j \in l_{i}} A_{c}^{\prime}\left(y_{i}, y_{j}\right)$.

For the image, combining all the energy functions of local region of image, we can receive MRF global energy model. 


$$
E_{G}(x)=\sum_{i} \frac{1}{c_{l}}\left[\sum_{i \in l_{i}} E_{i}\left(x_{i}\right)+\sum_{\forall i j \in l_{i}} E_{i j}\left(x_{i}, x_{j}\right)\right]=\sum_{i} \frac{1}{c_{l}} E_{l_{i}}\left(x_{i}\right)
$$

$i$ is ergodic the whole image, $l_{i} \in L$ is the local region of $i$, $\mathrm{R}$ is assemblage of local region, $c_{l}$ is neighborhood of pixel $l_{i}$ in local region $x_{i}$, ensuring energy of each pixel can be calculated only once. The global energy minimization problem of image segmentation on MRF is converted to global optimizing problem of local convergence. Local region is overlap, so the energy optimization of local region is converted to the neighborhood region. It ensure the smooth of image segmentation. At the same time, local structure of image, such as boundaries etc, can be kept efficient. So, for the problem of image segmentation, as global minimization energy problem of MRF, can be represented as:

$$
Y^{*}=\underset{x}{\arg \min } E_{G}(X)=\underset{x}{\arg \min } \sum_{i} \frac{1}{c_{l}} \underset{x_{i}}{\arg \min } E_{l_{i}}\left(x_{i}\right)
$$

\section{LBP Algorithm based on Minimizing Energy of Local Region}

Efficient optimization algorithm of image processing based on minimizing energy, such LBP algorithm [11, 12], graph cut algorithm [13, 14] etc., has been applied widely. LBP algorithm is a kind of iterating algorithm. The information on the node of MRF model is paralleled transmitted until convergence. In the standard LBP algorithm, the information of transmission is described by each single pixel. So it cannot sufficiently transmission the local region character of image. Information transmission of local region in LBP algorithm bring more local statistical characteristic of image. It raises the information of transmission message in LBP algorithm. Figure 2 shows the transmission of local region energy.

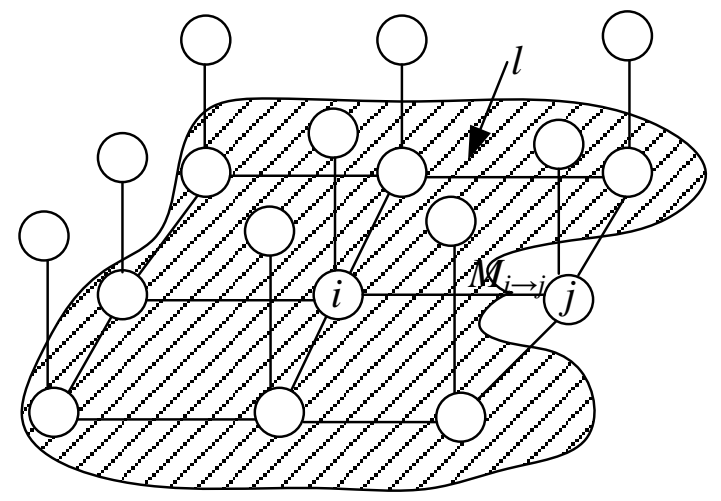

Figure 2. Local Regional Energy Transmit Sketch Map

The LBP algorithm based on local energy minimum:

$$
\begin{gathered}
m_{i \rightarrow j}^{t}\left(x_{j}\right)=\underset{x_{i}}{\min }\left(\sum_{i \in N(i) \backslash j} E_{i}\left(x_{i}\right)+E_{i j}\left(x_{i}, x_{j}\right)+\sum_{\substack{i j \in l_{i} \\
k \in N(i) \backslash j}} m_{\substack{t-1 \\
k \rightarrow i}}^{\left.\left.t x_{i}\right)\right)}\right. \\
y_{i}^{*}=\underset{x_{i}}{\arg \min } E_{L}\left(x_{i}\right)=\underset{x_{i}}{\arg \min }\left(\sum_{i \in l_{i}} E_{l_{i}}\left(x_{i}\right)+\sum_{i j \in l_{i}} E_{i j}\left(x_{i}, x_{j}\right)\right)
\end{gathered}
$$

(7) is updating message of formula in modified LBP algorithm. $m_{i \rightarrow j}^{t}\left(x_{j}\right)$ is message from node $i$ to node $j$ in local region in $t$ time iterations. The message is confirmed by local region energy of node $i . N(i) \backslash j$ is neighborhood node set of $i$, excepting node $j$. (8) is estimated formula. The label of node $i$ is received by the estimation of minimizing local region energy. 
During transmitting process of algorithm, although a larger local region bring more statistic character of local region, the calculation also increase. Complexity operation is $O\left(N k^{n-1} T\right) \cdot k$ is sum of label which is probably corresponded to each pixel. $T$ is number of iteration times. $\mathrm{n}$ is neighborhood number of node $i . N$ is pixel number of image.

Here two-way technology which could reduce the amount of information and belief will not affect the influence the result of image segmentation is proposed. First divide node into two sets $\mathrm{A}$ and $\mathrm{B}$, as Figure 3 shows:

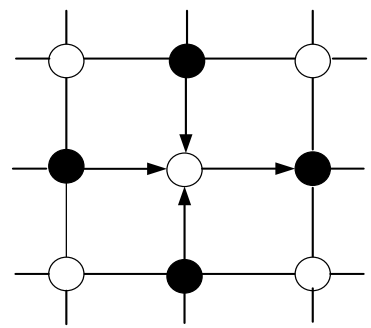

(a) filled-in circles pass Messages to empty circles

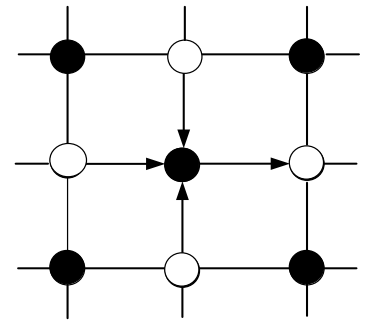

(b) empty circles pass messages to filled-in circles

Figure 3. Message Transmit Model

The solid circle represents node $A$, and hollow circle represents node $B$. So, there are two ways to translate message: $A \rightarrow B$ (Figure 3 (a)) $B \rightarrow A$ (Figure 3 (b)). During iteration of each time, just update one certain message. If update node message in $A$ during $t$ time iterations, we can calculate node information of $t+1$ time iterations in $B$, and we just need to update the node message of $t+2$ time iteration in $A$. Cycle this step.

Segmentation algorithm of local region minimizing energy is as: Firstly establish MRF model of segmenting image. Then estimate parameter $\mu_{k}, \sigma_{k}$ of GMM model, and calculate energy of each local region. Use LBP algorithm proposed in this paper to transmit information of local region to neighborhood region. And estimate the label of pixel by adopting the method of local region minimizing energy. Iterative operate until global convergence or reaching the maximum time of iteration, and received segmentation result. The detailed process of algorithm is as follows:

(1) Initialize algorithm. Prior parameter ${ }_{\beta}$ in MRF model is defined by experiment. Parameter $\mu_{k}, \sigma_{k}$ in GMM model initialize randomly.

(2) Estimate parameter $\mu_{k}, \sigma_{k}$ in GMM model by EM algorithm.

(3) Using the prior parameter $\beta$ in MRF model and parameter $\mu_{k}, \sigma_{k}$ estimated in GMM model, to beforehand segment image by MAP criterion $Y^{\prime}=\arg \max P\left(Y \mid X, \beta, \mu_{k}, \sigma_{k}\right)$.

(4) For $i=1$ to $N$, transmit message from local region.

(1) Calculate local region energy of node $x_{i}$ by (4), and transmit to neighborhood region by updating message regulation in (7);

(2) Estimate label $y_{i}{ }^{(n)}$ by MAP criterion in (8).

(5) Calculate global energy $E_{G \text { lobal }}^{(n)}(X)$ of MRF by (5).

(6) If $\left|E_{G \text { lobal }}^{(n)}(X)-E_{G \text { lobal }}^{(n-1)}(X)\right| \leq \varepsilon$, then $Y^{*}=Y^{(n)}$, receive the final segmentation result; otherwise, return to (4) to for the next iteration. 


\section{Image Segmentation and Experimental Analysis}

The experiment research on artificial noise image $(300 \times 300)$ and Concrete CT image $(400 \times 300)$ will be segmented by our algorithm, standard LBP algorithm, MMD, Gibbs, ICM. Segmentation parameters $\beta$ were given by experiment.

Experiment 1 Artificial Noise Image Segmentation. Respective defining 4 class mean value of artificial noise image is 50,100,150,200, and variance $\sigma_{k}=900$, pixels $(300 \times 300)$, as show figure 4. The image is hypothesis GMM model. Adopting LBP algorithm, MMD, Gibbs, ICM segment image in 4 classes on experiment. Result of segmentation is as show figure 4b-4i. Because of using local region information of image, segmentation results are better inhibition noise of image. So result of segmentation better than the result of standard of algorithm. Validity of algorithm use error rate to judge. Error rate as follows:

$$
r_{M C}=\frac{1}{N} \sum_{i}\left(1-\delta\left(x_{i}-x_{i}^{\text {true }}\right)\right)
$$

$\delta(*)$ Is Dikla function, $x_{i}^{\text {true }}$ is true label of pixel of $\mathrm{i}$.

Table 1 is error rate of various algorithm and operation time. The iteration and operation time of algorithms is corresponding to minimum error rate. From the date we can conclude, although operation time is a little more than others, our algorithm is fewer number of iteration and error rate of image segmentation is reduced. Result of segmentation better than others.

Table 1. Artificial Noise Image Different Algorithm Compares

\begin{tabular}{ccccccc}
\hline \hline \multirow{2}{*}{ Algorithm } & \multicolumn{2}{c}{$\mathrm{r}_{\mathrm{MC}} / \%$} & \multicolumn{2}{c}{ Iteration } & \multicolumn{2}{c}{ Operation Time/s } \\
\cline { 2 - 7 } & $\begin{array}{c}\text { Standard } \\
\text { algorithm }\end{array}$ & $\begin{array}{c}\text { Our } \\
\text { algorithm }\end{array}$ & $\begin{array}{c}\text { Standard } \\
\text { algorithm }\end{array}$ & $\begin{array}{c}\text { Our } \\
\text { algorithm }\end{array}$ & $\begin{array}{c}\text { Standard } \\
\text { algorithm }\end{array}$ & $\begin{array}{c}\text { Our } \\
\text { algorithm }\end{array}$ \\
\hline ICM & 0.009364 & 0.006522 & 8 & 6 & 0.728 & 5.316 \\
Gibbis & 0.009881 & 0.005589 & 115 & 130 & 19.721 & 71.449 \\
MMD & 0.010959 & 0.007667 & 219 & 184 & 6.502 & 24.719 \\
LBP & 0.007378 & 0.004967 & 21 & 7 & 2.18 & 4.003 \\
\hline \hline
\end{tabular}

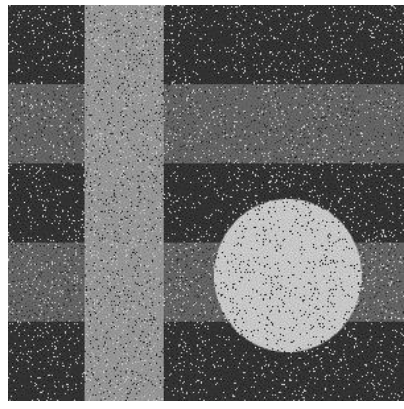

Figure 4. (a) Original Image 

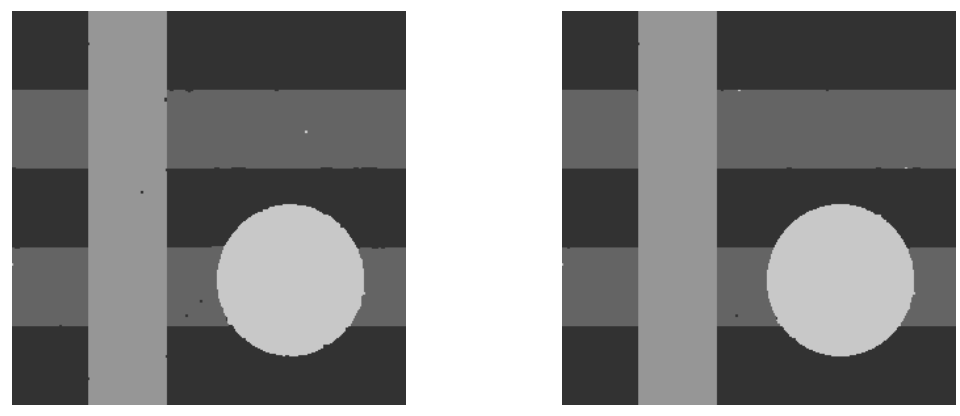

Figure 4. (b)ICM(standard $\beta=2.1$ )
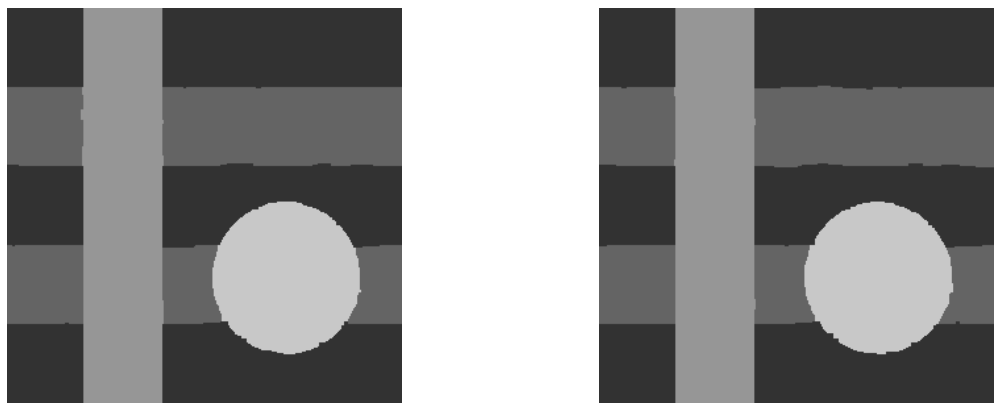

Figure 4. (d)Gibbs (standard $\beta=2.0$ )

Figure 4. (e) $\operatorname{Gibbs}($ our $\beta=5.8)$
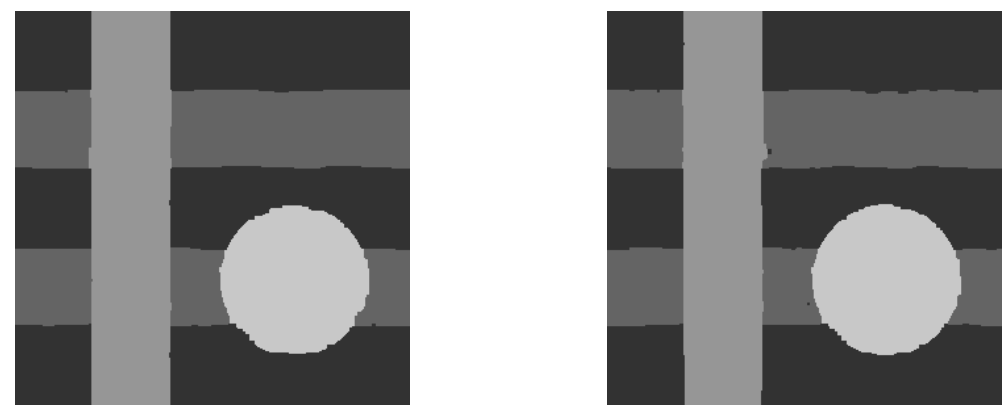

Figure 4. (f)MMD(standard $\beta=2.7$ )

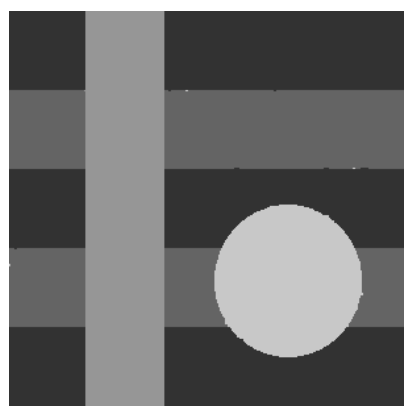

Figure 4. (g) Gibbs(our $\beta=6.5$ )

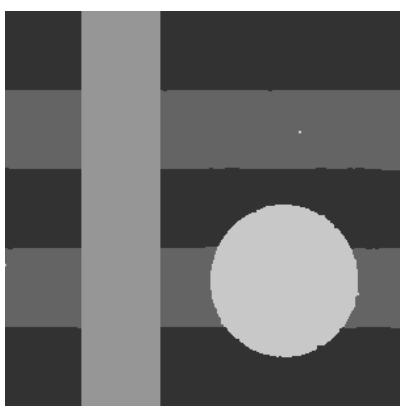

Figure 4. (h) LBP (standard $\beta=2.1$ )

Figure 4. (i) LBP (our $\beta=4.8$ )

Figure 4. Artificial Noise Image Segmentation

Experiment 2 Concrete CT Image Segmentation. Concrete is a kind of special natural defect material, which is consisted of graded aggregate, cement, mortar and 
holes, the inner construction is complicated, it contains multi-scale and unique physical and mechanical properties, and widely used in the construction industry uneven material [15]. Concrete CT image can be segmented 3 classes. Result as show Figure 5. Contrasting our algorithm and standard algorithm from segmentation result, result of our algorithm is better. Another, because our algorithm contain more local image information, so not only good segmentation result can be obtain, but efficient reduce redundancy iteration and operation time of algorithm. Iteration time of algorithm is contrast as show Table 2 .

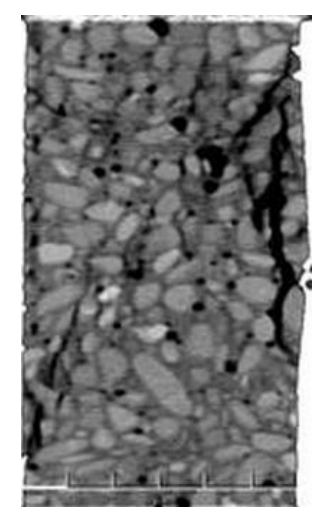

Figure 5. (a)12.7MPa original image

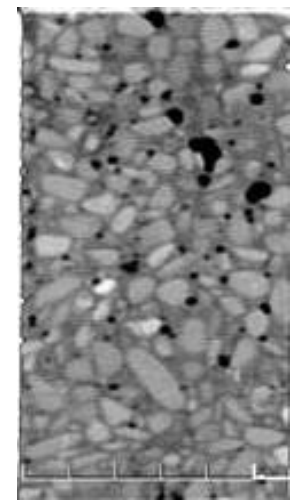

Figure 5. (d)34.89MPa original image

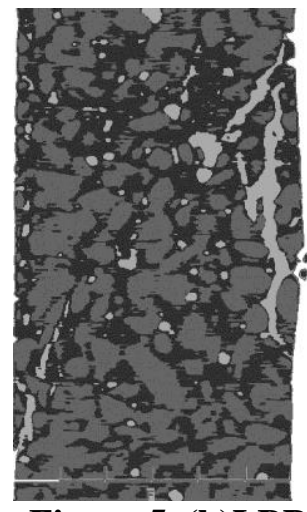

Figure 5. (b)LBP
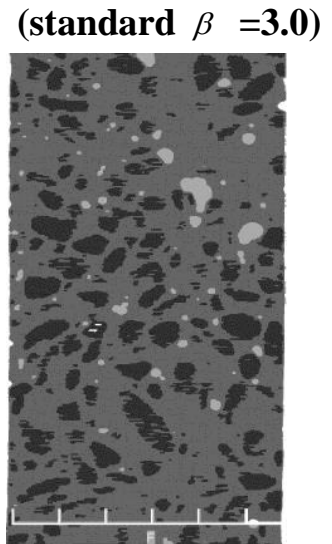

Figure 5. (e)LBP

(standard $\beta=4.2$ )

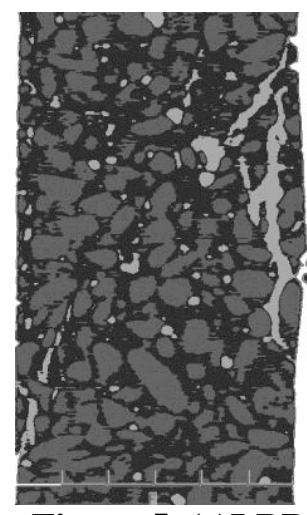

Figure 5. (c)LBP

(our $\beta=2.1$ )

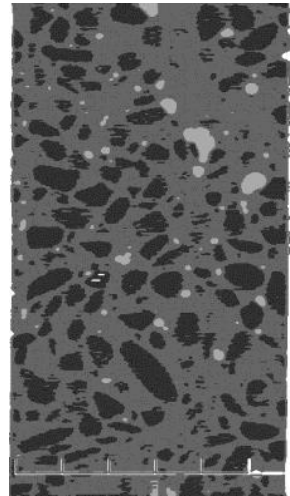

Figure 5. (f)LBP

(our $\beta=1.8$ )

Figure 5. Concrete CT Image Segmentation

Table 2. Concrete CT Image Segmentation Time Compares

\begin{tabular}{ccccc}
\hline \hline \multirow{2}{*}{ Algorithm } & \multicolumn{2}{c}{ Iteration } & \multicolumn{2}{c}{ Operation Time/s } \\
& Figure 5. (a) & Figure 5. (d) & Figure 5. (a) & Figure 5. (d) \\
\hline standard Algorithm & 49 & 38 & 24.548 & 9.128 \\
Our Algorithm & 11 & 12 & 7.843 & 4.950 \\
\hline \hline
\end{tabular}

Result of segmentation shows our algorithm not only can good segment smooth regions of the image, but better on texture region(mortar) and noise region than standard algorithm. Our algorithm is also faster. 


\section{Conclusions}

Image segmentation algorithm based on minimizing the energy of local region is proposed apply on concrete CT image segmentation. Our algorithm establishes a segmentation model of local interaction region based on Pairwise MRF, combines convergence of local region energy with minima of global energy. Result of segmentation shows our algorithm efficient restrain image noise and texture mutations effects on image segmentation. From concrete CT image segmentation, we can see the materials distribution and the changing process of cracks or holes with the stress distribution clearly, which simplified the following CT statistical analysis and provide an important method for reaching real meso-structure of concrete's finite element network.

\section{Acknowledgements}

This work was financially supported by National Nature Science Foundation of China (Grant No.51209167), Nature Science Foundation of Shaanxi Province(Grant No. 2013JQ7024), ShaanXi Educational Committee (Grant No.12JK0520), and Xi'an urban and rural construction commission under (Grant No.SJW201223).

\section{References}

[1] R. M. Haralick and L. G. Shapiro, "Survey: image segmentation techniques", Computer Vision, Graphics, and Image Process, vol. 29, (1985), pp. 100-132.

[2] B. Potetz and T. S. Lee, "Efficient belief propagation for higher order cliques using linear constraint nodes", Computer Vision and Image Understanding, vol. 112, no. 1, (2008), pp. 39-54.

[3] M. Tanaka and M. Okutomi, "Locally adaptive learning for translation-variant MRF image priors", IEEE Conference on Computer Vision and Pattern Recognition, IEEE Computer Society, (2008). pp. 1-7.

[4] B. Potetz, "Efficient belief propagation for vision using linear constraint nodes", Proceedings of IEEE international conference on computer vision and pattern recognition, IEEE Computer Society, (2007), pp. 1-8.

[5] T. Yan, L. Ji-jun, X. Yu-bo, et al., "Extraction of transition region and image segmentation based on local fuzzy variance", Journal of Infrared and Millimeter Waves, vol. 26, no. 5, (2007), pp. 386-389.

[6] W. Xiao-feng, Z. Xiao-ping, "A new localized superpixel Markov random field for image segmentation", Proceedings of 2009 IEEE Conference Multimedia and Expo, IEEE, (2009), pp. 642-645.

[7] J. Jian-hua, J. Li-cheng and C. Xia, "Image segmentation via mean shift and loopy belief propagation", Wuhan University Journal of Natural Sciences, vol. 15, no. 1, (2010), pp. 43-50.

[8] C. Jian-sheng, S. Guang-da, H. Jin-ping, et al., "Face image relighting using locally constrained global optimization", Proceedings of European Conference on Computer Vision, Springer, (2010), pp. 44-57.

[9] S. Z. Li, "Markov random field modeling in computer vision", Sprinter-Verlag, (2001).

[10] J. Yedidia, W. Freeman and Y. Weiss, "Generalized belief propagation", Proceedings of advances in neural information processing Systems, MIT Press, (2000), pp. 689-695.

[11] S. Jian-bo and J. Malik, "Normalized cuts and image segmentation", IEEE Transactions on Pattern Analysis and Machine Intelligence, vol. 22, no. 8, (2000), pp. 888-905.

[12] X. Lan and R. Stefan, "Efficient belief propagation with learned Higher-order Markov Random Fields", Processings of ECCV, vol. 2, (2006), pp. 269-282.

[13] E. Sharon, M. Galun, D. Sharon, et al., "Hierarc;hy and adaptivity in segmenting visual scenes", Nature, vol. 442, no. 7104, (2006), pp. 810-813.

[14] Z. Qiang and W. Yan, "Wavelet Markov random field based on context and hidden class label for SAR image segmentation", Journal of Electronic \&Information Technology, vol. 30, no. 1, (2008), pp. 211-215.

[15] Z. Liang, L. Jun, X. Sheng-Jun, et al., "Multiscale belief propagation on concrete CT image fast segmentation ", International Journal of Grid and Distributed Computing, vol. 6, no. 6, (2013), pp. 13-22. 


\section{Authors}

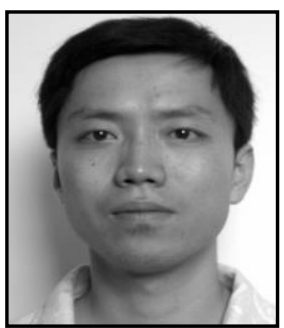

Liang Zhao, was born 1980. He completed his Ph.D. degree in Xi'an University of Architecture and Technology .He research focus on computer control, measure and detection technique.

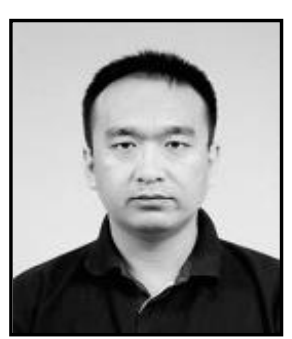

Sheng-jun $\mathrm{Xu}$, received $\mathrm{Ph} . \mathrm{D}$ degree from Xi'an JiaoTong University. His research interests include image processing, computer vision, and pattern recognition.

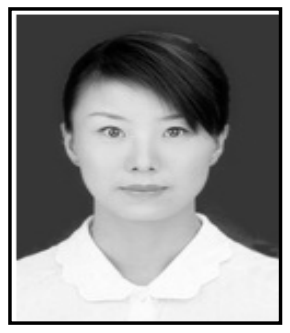

Jun Lu, received M.S degree from XiDian University. Her research interests in computer control, information system.

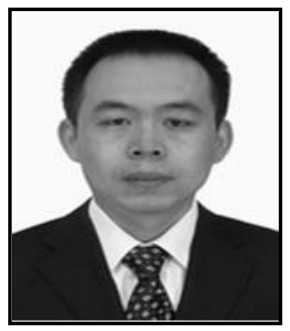

Deng-feng Chen, completed his Ph.D. degree in Northwestern Polytechnical University. He mainly focus research on computer detection technique, control engineering. 